\title{
Identification of a conserved var gene in different Plasmodium falciparum strains
}

\author{
Sandra Dimonte ${ }^{1 \dagger}$, Ellen I. Bruske ${ }^{1 \dagger}$, Corinna Enderes ${ }^{1}$, Thomas D. Otto ${ }^{2,3}$, Louise Turner ${ }^{4,5}$, Peter Kremsner ${ }^{1}$ \\ and Matthias Frank ${ }^{1 *}$ (D)
}

\begin{abstract}
Background: The multicopy var gene family of Plasmodium falciparum is of crucial importance for pathogenesis and antigenic variation. So far only var2csa, the var gene responsible for placental malaria, was found to be highly conserved among all P. falciparum strains. Here, a new conserved 3D7 var gene (PF3D7_0617400) is identified in several field isolates.

Methods: DNA sequencing, transcriptional analysis, Cluster of Differentiation (CD) 36-receptor binding, indirect immunofluorescence with PF3D7_0617400-antibodies and quantification of surface reactivity against semi-immune sera were used to characterize an NF54 clone and a Gabonese field isolate clone (MOA C3) transcribing the gene. A population of 714 whole genome sequenced parasites was analysed to characterize the conservation of the locus in African and Asian isolates. The genetic diversity of two var2csa fragments was compared with the genetic diversity of 57 microsatellites fragments in field isolates.
\end{abstract}

Results: PFGA01_060022400 was identified in a Gabonese parasite isolate (MOA) from a chronic infection and found to be 99\% identical with PF3D7_0617400 of the 3D7 genome strain. Transcriptional analysis and immunofluorescence showed expression of the gene in an NF54 and a MOA clone but CD36 binding assays and surface reactivity to semiimmune sera differed markedly in the two clones. Long-read Pacific bioscience whole genome sequencing showed that PFGA01_060022400 is located in the internal cluster of chromosome 6. The full length PFGA01_060022400 was detected in 36 of 714 P. falciparum isolates and 500 bp fragments were identified in more than 100 isolates. var2csa was in parts highly conserved $\left(H_{e}=0\right)$ but in other parts as variable $\left(H_{e}=0.86\right)$ as the 57 microsatellites markers $\left(H_{\mathrm{e}}=0.8\right)$.

Conclusions: Individual var gene sequences exhibit conservation in the global parasite population suggesting that purifying selection may limit overall genetic diversity of some var genes. Notably, field and laboratory isolates expressing the same var gene exhibit markedly different phenotypes.

Keywords: Malaria, var genes, Genetic diversity, Microsatellites, Recombination, VSA, PfEMP1, rifin, stevor

\footnotetext{
*Correspondence: matthias.frank@praxis-frank-tuebingen.de

†Sandra Dimonte and Ellen I. Bruske contributed equally to this work

${ }^{1}$ Institute of Tropical Medicine, University of Tuebingen, Wilhelmstr. 27, 72074 Tuebingen, Germany

Full list of author information is available at the end of the article
}

\begin{abstract}
Background
The most virulent form of malaria is caused by Plasmodium falciparum [1]. The virulence of $P$. falciparum is a consequence of adhesion of infected red blood cells (iRBCs) to different host endothelial receptors [2]. This process is primarily mediated by a polymorphic protein family collectively referred to as $P$. falciparum membrane protein 1 (PfEMP1) [2-4]. Expression of PfEMP1 variants that exhibit binding to chondroitin sulfate $[5,6]$ in the
\end{abstract}


placenta or to the endothelial protein $C$ receptor (EPCR) [7] has been associated with the development of placental malaria and cerebral malaria, respectively. PfEMP1 is encoded by the var gene family [8], a multicopy gene family with approximately 59-60 different genes per parasite genome [9]. Only one var gene is expressed in an individual parasite at a time, a process referred to as mutually exclusive expression [10]. Switching between the active var locus generates antigenic variation leading to immune escape of the parasites [11].

var genes can be grouped according to their distribution across the 14 P. falciparum chromosomes into subtelomeric or central and this position correlates with their promoter type [12]. 36 of the 59 var genes in the 3D7 genome strain are located in subtelomeric areas and have upstream (Ups)A, UpsB or UpsB/A type promoters, while 23 are located in central var clusters and have $\mathrm{Ups} C$ or UpsB/C type promoters. An individual var gene typically consists of exon 1 and exon 2 that encode for the extracellular and intracellular parts of the PfEMP1 protein, respectively. Exon 1 is hypervariable and consists of an $\mathrm{N}$-terminal segment, followed by a variable number of domain cassettes (DCs) that are composed of several Duffy Binding Like- (DBL) and Cysteine Rich Interdomain Region-(CIDR) domains [9]. DBL and CIDR domains are assembled of conserved sequence blocks that share similarities in all parasite strains as well as hypervariable sequence blocks [13]. The 60 var genes of an individual parasite are almost completely distinct from each other and different $P$. falciparum strains carry almost completely different var gene repertoires [14, 15]. This tremendous genetic diversity appears to be the result of the highly recombinogenic nature of the ATrich $P$. falciparum genome, the diversifying pressure of the immune system and the virtual absence of purifying selection on the var gene family. Indeed recombination events generate new var gene variants during meiosis and mitosis $[16,17]$. Furthermore binding phenotypes such as CD36 and EPCR binding appear to be primarily mediated by the tertiary structures of the CIDR domain and by hydrophobicity, characteristics that do not require an exact amino acid motif, thus allowing a high degree of sequence variation without affecting the function of the respective PfEMP1 domains [18].

Considering the high variability of the var gene family, it is remarkable that there is one var gene that is conserved among all P. falciparum strains. This gene, var2csa, [5] encodes the PfEMP1 VAR2CSA that binds to chondroitin sulfate A (CSA) in the human placenta and is primarily responsible for placental malaria during the first pregnancy [6]. Because of this unique binding phenotype and function, there appears to be positive selection pressure to maintain this var gene.
During a var gene transcription analysis of a field isolate from Gabon (called MOA), a DBL with 100\% sequence identity to the DBL of PF3D7_0617400 of the 3D7 reference genome [19] was identified. In this work, the MOA allele of the gene (PFGA01_060022400) is shown to be $99 \%$ identical with PF3D7_0617400. The gene was detected in 36 of 714 African and Asian parasite suggesting a selective advantage for parasites carrying this var gene.

\section{Methods}

\section{Parasite lines and cultures}

The MOA bulk culture was originally obtained from an chronic asymptomatic infection of a Gabonese individual as previously described $[19,20]$. A total of 19 clones were generated by limiting dilution in two independent cloning experiments $[19,20]$. The MOA C3 clone and the MOA D2 (PfGB01) [21] clone were generated in the first cloning experiment directly after tissue culture adaptation of the MOA bulk culture [19]. The NF54 A3 clone was generated from an NF54 bulk culture as described previously [22]. Parasite stocks in glycerolite of the NF54 A3 [22] laboratory strain, the culture adapted Gabonese MOA C3 [19] and $\triangle$ MOA D2 (PFGB01) [20] field isolates were thawed by slowly adding 5 drops of $12 \% \mathrm{NaCl}$ solution and a $5 \mathrm{ml}$ of $1.6 \% \mathrm{NaCl}$ solution. The stocks were then spun down, the supernatant was discarded and the parasites were used to inoculate an in vitro culture.

The isolates 5259, 12295, 5420, 12480, 5798, 3256, $3324,6022,6210$ were culture adapted from diagnostic specimens submitted for routine malaria diagnosis to the laboratory of the outpatient clinic of the Institute of Tropical Medicine in Tübingen, Germany. After conducting routine thick and thin blood smears, the remainder of the EDTA blood tube was centrifuged, the serum was separated and $500 \mu$ lerythrocyte pellet was used to inoculate $5 \mathrm{ml}$ culture medium. After successful culture adaptation, parasites were expanded into a $20 \mathrm{ml}$ culture and cryopreserved stocks as well as cell pellets were stored for future investigations. The $\triangle \mathrm{MOA}$ D2 parasite line has been previously described [20]. All P. falciparum isolates were cultivated at $5 \%$ haematocrit of $0^{+}$erythrocytes from a local cell bank. RPMI 1640 medium was completed with 10\% Albumax (Gibco), 25 mM HEPES Buffer, $2 \mathrm{mM} \mathrm{L}$-Glutamine and $0.05 \mathrm{mg} / \mathrm{ml}$ gentamicin (all PAA Laboratories). Parasites were incubated at $37^{\circ} \mathrm{C}$ in $90 \%$ nitrogen, $5 \%$ oxygen and $5 \%$ carbon dioxide. $\Delta$ MOA D2 was cultured in medium without blasticidin as previously described [20].

\section{DNA extraction}

Cell pellets for DNA extraction were stored at $-20{ }^{\circ} \mathrm{C}$. After thawing of the pellet, DNA was extracted using 
the QIAmp ${ }^{\circledR}$ DNA Blood Midi Kit (Quiagen, Cat. No. 51185) following the manufacturer's protocol. DNA content was measured by Nanodrop ${ }^{\circledR} 1000$ 3.7.1 (Nanodrop Technologies).

\section{Polymerase chain reaction (PCR) and agarose gel electrophoresis}

For qualitative PF3D7_0617400 PCR, a specific primer set was designed, spanning exon 1 from promoter until the intron (Additional file 1: Table S1). Overlapping fragments for the entire exon 1 were generated. PCR with the var2csa specific primers $10 \mathrm{~F}$ and $75 \mathrm{R}$ [5] was employed to amplify an approximately $1700 \mathrm{bp}$ fragment of var2csa. The first 40 BP of exon 1 of PF3D7_0617400 were characterized with the UpsC promoter primer. This primer was originally developed by Rottmann et al. [23] and has been previously evaluated on the MOA field isolate [19].

$5 \mu \mathrm{l}$ genomic DNA were mixed with $31 \mu \mathrm{l} \mathrm{H2O}, 0.4 \mu \mathrm{l}$ dNTPs, $3 \mu \mathrm{l} \mathrm{MgCl}, 5 \mu \mathrm{l}$ buffer, $2.5 \mu$ l forward primer, $2.5 \mu \mathrm{l}$ reverse primer, and $0.3 \mu \mathrm{l}$ Taq-polymerase to a final volume of $50 \mu \mathrm{l}$ per reaction. Standard PCR conditions were $94{ }^{\circ} \mathrm{C}$ for $3 \mathrm{~min}, 40 \times\left(94{ }^{\circ} \mathrm{C}\right.$ for $10 \mathrm{~s}$, $54{ }^{\circ} \mathrm{C}$ for $30 \mathrm{~s}$, $72{ }^{\circ} \mathrm{C}$ for $30 \mathrm{~s}$.), $72{ }^{\circ} \mathrm{C}$ for $3 \mathrm{~min}$. For the primers pairs $\mathrm{P} 3$, $\mathrm{P} 5$ and $\mathrm{P} 6$ annealing and elongation temperatures were adjusted according to primer melting temperature and fragment length. Individual primer specific PCR conditions can be obtained from the author upon request. PCR fragments were separated using gel electrophoresis with a $1 \%$ agarose gel, 80-120 V current.

\section{Sorbitol synchronization, RNA extraction and CDNA synthesis}

$20 \mathrm{ml}$ parasite cultures were used for RNA extraction. The culture was pelleted, washed several times with $1 \times$ PBS and the erythrocytes were lysed with $0.02 \%$ saponin. The pellet was then washed 3 times with $1 \times$ PBS and resolved in $750 \mu$ of Trizol ${ }^{\circledR}$ LS Reagent (Invitrogen). The samples were stored at $-20^{\circ} \mathrm{C}$ until further processing.

For RNA extraction, $0.2 \mathrm{ml}$ chloroform were added to $750 \mu \mathrm{l}$ of thawed Trizol-lysate, shaken vigorously for $15 \mathrm{~s}$ and left at room temperature for $10 \mathrm{~min}$. A $15 \mathrm{~min}$ centrifugation step at 12,000 $\mathrm{g}$ at room temperature followed for phase separation. RNA was extracted from the aqueous phase with the PureLink ${ }^{\mathrm{TM}}$ RNA Mini Kit (ambion by Life Technologies) according to the manufacturer's protocol. RNA content was measured by Nanodrop ${ }^{\circledR}$ 1000 3.7.1 (Nanodrop Technologies). All samples were treated with DNAse $\mathrm{I}^{\circledR}$ (Invitrogen) according to the manufacturer's protocol to remove any remaining DNA. cDNA was synthesized with random primers and Superscript II Reverse Transcriptase ${ }^{\circledR}$ (Invitrogen) according to the manufacturer's protocol. cDNA was tested for absence of DNA contamination by evaluation of proper splicing of the gene PFD1155w by PCR with the primer 5'GCAGGGAAAGGTTTTTCAAG3' and the reverse primer $5^{\prime}$ AAAGCTGAATCTTGGCCCGTT $3^{\prime}$ as described elsewhere [22].

\section{Var gene transcription analysis}

\section{cDNA DBL cloning}

For analysis of specific var gene expression in the MOAC3 clone, the active var locus was identified by cloning cDNA var PCR fragments obtained with universal primers [24] followed by sequencing. The experiment was performed in two biological replicates directly after the original limiting dilution experiment of the MOA bulk culture [19].

\section{Quantitative real-time $P C R$}

For quantitative RT-PCR reactions of the NF54 A3 strain, we employed the primer set of Salanti et al. [5] with the modifications as previously described [10, 19, 25] . For quantitative RT-PCR of the MOA field isolate the MOA primers C3_C3, D2_D2 and D5_D5 were used [19]. All reactions included five housekeeping genes as controls: seryl-tRNA synthetase (PF3D7_0717700), fructose bisphosphate aldolase (PF3D7_1444800), actin (PF3D7_1246200), arginyl-tRNA synthetase (PF3D7_1218600) and glutaminyl-tRNA synthetase (PF3D7_1331700) [25]. Reactions were performed at a final primer concentration of $0.25 \mu \mathrm{M}$ using SensiMix SYBR No-ROX Kit (Bioline, QT650-05) in $20 \mu \mathrm{l}$ reactions, measured in Corbett Research Rotorgene 3000 $\left(95{ }^{\circ} \mathrm{C}\right.$ for $3 \mathrm{~min} / 95^{\circ} \mathrm{C}$ for $15 \mathrm{~s}, 54{ }^{\circ} \mathrm{C}$ for $30 \mathrm{~s}, 68{ }^{\circ} \mathrm{C}$ for $30 \mathrm{~s}, 40$ cycles $/ 68^{\circ} \mathrm{C}$ for $1 \mathrm{~min}$ ). The same threshold was used for all analysis. var gene copy numbers were determined relatively to PF3D7_1218600, using the $\Delta \Delta C T$ method [5].

\section{Targeted Sanger sequencing}

For PCR fragment-sequencing, the PCR product was purified from an agarose gel or the PCR solution, using the Quiagen PCR purification kit according to the manufacturer's protocol. Sequencing PCR was performed at a final reaction volume of $10 \mu \mathrm{l}$ per reaction, containing $1 \mu$ BigDye, $2 \mu$ reaction buffer, $2.5 \mu$ forward or reverse primer, purified water and purified PCR product according to DNA concentration. Sequencing PCR conditions were: $94{ }^{\circ} \mathrm{C}$ for $10 \mathrm{~s}, 50{ }^{\circ} \mathrm{C}$ for $5 \mathrm{~s}, 60^{\circ} \mathrm{C}$ for 4 min, back cycle to beginning: $25 \times$. After PCR, all samples were cleaned up by Sephadex agarose column centrifugation. Sanger sequencing was performed in the Applied Biosystems ABI Prism 3130xl Genetic Analyzer. 


\section{Whole genome sequencing}

Long-read Pacific Biosciences whole genome sequencing of the MOA D2 (PFGB01) and 5798 (PFTG01) parasite lines parasite was performed as previously described [21].

\section{Fragment analysis}

57 microsatellites (MS) distributed over the 14 chromosomes of $P$. falciparum were analysed by multiplex fragment analysis (Additional file 2: Table S2). DNA of all freshly culture adapted field isolates, the MOA C3 strain as well as the NF54 A3 were analysed. MS amplification and fragment length analysis were done as previously described [26]. In four strains, approximately $30 \%$ of MS showed 2 amplification products/peaks (5798, 3324, $5420,12480)$. The higher peak was arbitrarily chosen as the representative allele for the respective marker.

\section{Calculation of expected heterozygosity}

The expected heterozygosity $\left(\mathrm{H}_{\mathrm{e}}\right)$ was calculated using the formula $\mathrm{H}_{\mathrm{e}}=[\mathrm{n} /(\mathrm{n}-1)][1-\Sigma \mathrm{pi} 2]$, where $\mathrm{n}$ is the total number of alleles at a distinct locus and reflects the proportion of the individual alleles as described previously [27]. A new allele was defined as a difference of more $>3$ bp between PCR fragments [26]. The same criterion was applied for allele definition of the var2csa fragments used for the $\mathrm{H}_{\mathrm{e}}$ calculation of the var2csa gene.

\section{Assembling of sequences}

Sequences were assembled and aligned using SeqScape software by Applied Biosystems.

\section{Comparing sequences}

The long read sequences were compared using the ACT software by Artemis [28]. To compare the var gene sequences with the var gene database, a megablast (-e 1e-6 -m 8 -a 8 -v 15000 -b 15000 -F F) against the normalised data base from Otto et al. [15] was done (varDB. Normalised.3 kb.nt.noVARx_noExon2.fasta.gz)-ftp ftp:// ftp.sanger.ac.uk/pub/project/pathogens/Plasmodium/ falciparum/PF3K/varDB/NormalisedDataset/. To look for SNP around the conserved locus on chromosome 6, SNP were called with mpileup : (bcftools mpileup -r Pf3D7_06_v3 -f Pf3D7_06_v3.fasta \$x.bam | bcftools call -cv -Ov - ploidy 1 -o SNP.var.\$x.vcf).

Antibody preparation and Immunofluorescence assay (IFA) The recombinant CIDRa2.1 domain of PF3D7_0617400 (MAL6P1.252) was produced at the Statens Seruminstitut, Cophenhagen, Denmark as described in [18] and used to immunize mice. Blood of immunized mice was sent to the Institute of Tropical Medicine, Tübingen, Germany. Mouse sera containing IgG $\alpha$ PF3D7_0617400 mouse secondary antibody were depleted from RBCs by filtering through a MN $615 \frac{1}{4}$ filter. IgG was purified using Protein G Spin Columns from Thermo Scientific according to the manufacturer's protocol.

For IFA, very thin blood smears of parasite cultures were fixated in $-20{ }^{\circ} \mathrm{C}$ cold $100 \%$ methanol for $5 \mathrm{~min}$ and then stored at $-20{ }^{\circ} \mathrm{C}$ until further use. IFA was performed following a modified protocol as previously described [29].

After a 5 min rehydration step in $1 \times$ PBS, slides were incubated for 1-2 $\mathrm{h}$ with anti CIDRa2.1 PF3D7_0617400 (diluted 1:50 in 1xPBS/1\%BSA). The slides where then washed $3 \times$ with $1 \times P B S$ and incubated for $1 \mathrm{~h}$ with Alexa488 coupled mouse sera IgG $\alpha$ PF3D7_0617400 mouse secondary antibody (not diluted, $0.44 \mathrm{mg} / \mathrm{ml}$ ). After another $3 \times$ washing with $1 \times$ PBS, slides were stained with Hoechst 33342 (diluted 1:1000) for $30 \mathrm{~min}$. Slides were mounted over night with MOWIOL-488 and viewed through $100 \times$ oil immersion lens at a fluorescent microscope.

\section{CD36 receptor binding selection}

Human melanoma C32 (ATCC1, CRL-1585 ${ }^{\mathrm{TM}}$ ) cells were used to select CD36 binding infected red blood cells (iRBCs) as previously described [20].

\section{Flow cytometry analysis}

Flow cytometry analysis of MOA C3, NF54 A3 and $\Delta$ MOA D2 with serum of the MOA individual was performed as described in [20].

\section{Results}

Identification of the 3D7 var gene PF3D7_0617400 in a field isolate from Gabon

The $P$. falciparum field isolate MOA was obtained from an asymptomatically infected Gabonese individual [19, 20, 30]. After tissue culture adaption, a total of 19 clones were generated by limiting dilution and the transcribed var genes were determined by cDNA DBL cloning. In the MOA clone C3 [19], the transcribed DBL (MOAC3) was found to be $100 \%$ identical with the DBL of the 3D7 var gene PF3D7_0617400 (previously annotated as MAL6P1.252/PFF0845c). To determine the extent of sequence homology, a primer set (Additional file 1: Table S1) spanning the promoter region and the entire exon one (bp 0-6271) of PF3D7_0617400 was designed based on the 3D7 genome sequence. All PCR fragments obtained from MOA C3 genomic DNA were characterized by Sanger sequencing and comparison of the assembled MOA C3 sequence (GenBank accession number MG507307) with the 3D7 reference sequence showed that the sequences were $99-100 \%$ identical (Fig. 1). The only difference consisted of a small 194 bp insertion 

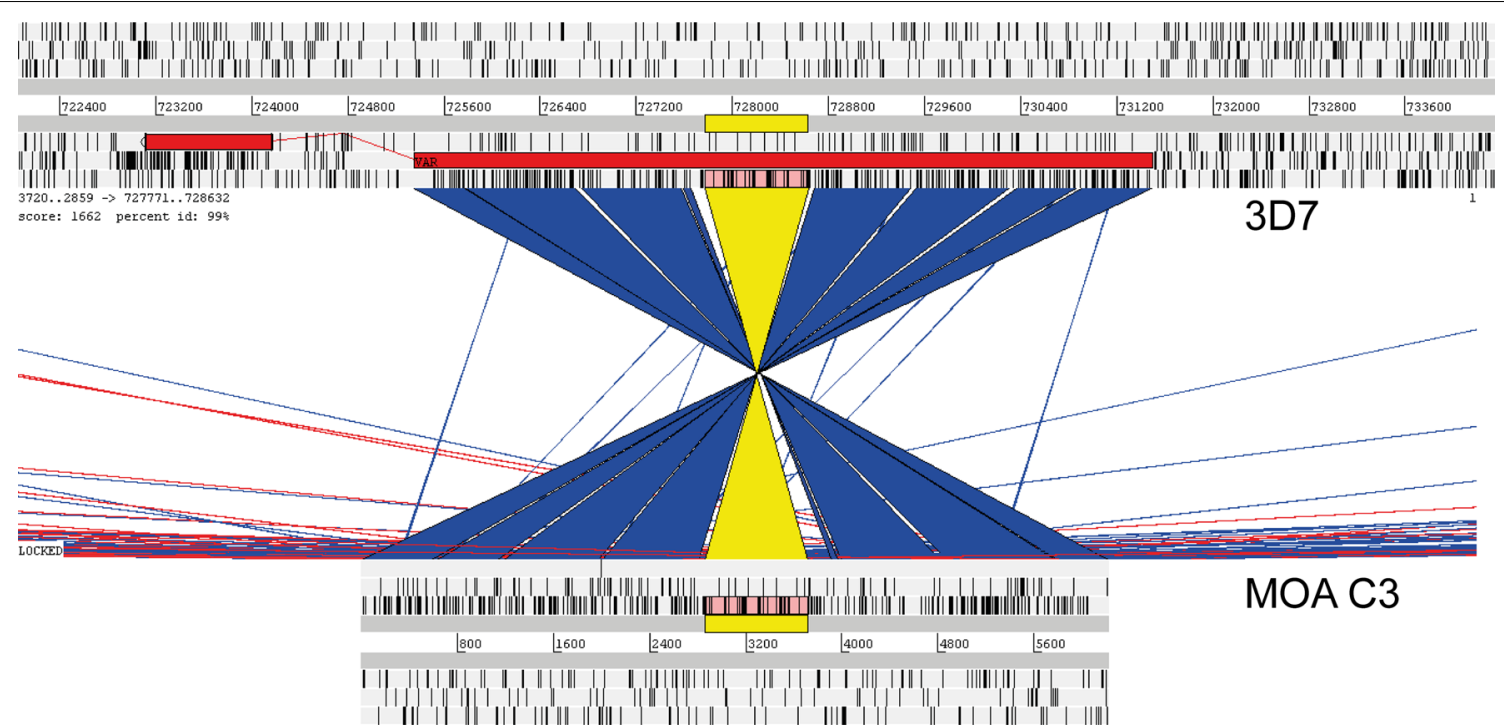

MOA C3

Fig. 1 Screenshot of ACT program comparing the assembled sequence of PF3D7_0617400 of the field isolate clone MOA C3 with the reference strain 3D7. The upper part of the figure depicts the 3D7 reference sequence. The lower part shows the MOA C3 sequence. Blue and yellow $=$ sequence identical. White: missing sequence (insertion). For orientation, the sequence next to the insertion that differs between MOAC3 and 3D7 is coloured in yellow. The additional small white areas represent homopolymer runs. Visual examination of direct alignments of the homopolymer runs revealed no differences in these areas (data not shown)

between base pairs 3722 and 3871 of the MOA C3 gene (3721 and 3914 of the 3D7 reference sequence).

\section{MOA C3 and NF54 A3 both express the same var gene but have different cytoadhesion and surface recognition signal phenotypes}

To determine if the entire open reading frame (ORF) of PF3D7_0617400 was transcribed in MOA C3, cDNA was analysed by qualitative and quantitative PCR. PCR transcripts were obtained with 6 primers spanning exon 1 of PF3D7_0617400 (Fig. 2a) showing that the full length RNA of the gene was transcribed and quantitative PCR (qPCR) with DBL specific primers showed that the transcriptional signal for PF3D7_0617400 was in the range of a dominantly transcribed var gene in MOA C3 (Fig. 2b). During previous investigations the NF54 clone A3 had been generated that transcribed PF3D7_0617400 in a very stable fashion [22]. NF54 A3 was therefore rethawed and brought into tissue culture. Consistent with the previously reported low off rate of PF3D7_0617400, it was still the dominantly transcribed var gene in this regrown culture (Fig. 2c).

To assess the binding phenotype of PF3D7_0617400, CD36 binding assays with the clones NF54 A3 and MOA C3 were conducted on human melanoma cells expressing CD36. NF54 A3 exhibited CD36 binding even without prior selection and the CD36 binding capacity increased strongly after 2 additional rounds of panning (Fig. 3a). In contrast, MOA C3 did not exhibit significant CD36 binding prior to selection and even after 4 rounds of panning CD36 binding increased only marginally (Fig. 3b). NF54 A3 transcriptional analysis after CD36 selection showed a strong increase in PF3D7_0617400 transcription, showing that it mediated the phenotype (Additional file 3: Fig. S1a). However, transcriptional quantification of PF3D7_0617400 in MOA C3 showed no increase in transcriptional signal (Additional file 3: Fig. S1b). To quantify overall surface PfEMP1 expression in CD36 selected NF54 A3 and MOA C3 clones, FACS analysis with serum from the semi-immune MOA [20] patient was conducted. CD36 selected NF54 A3 parasites displayed a strong FACS signal with the semi-immune serum, clearly showing that the serum detected the expressed PfEMP1 on the surface of the iRBCs (Fig. 3c). In contrast, CD36 selected MOA C3 parasites only displayed a low FACS signal with semi-immune serum.

To determine if the corresponding PfEMP1 was indeed expressed in both strains, immunofluorescence assays with an antibody against the CIDRa2.1 of PF3D7_0617400 were conducted [18] . An immunofluorescence signal was detected in MOA C3 (Fig. 4a) and in the NF54 A3 infected erythrocytes (Fig. 4b) [20] showing that both strains indeed expressed the same PfEMP1 [20]. Together the data suggested that although both strains expressed the same var gene, there was a difference in PfEMP1 display between the NF54 and MOA parasites. 

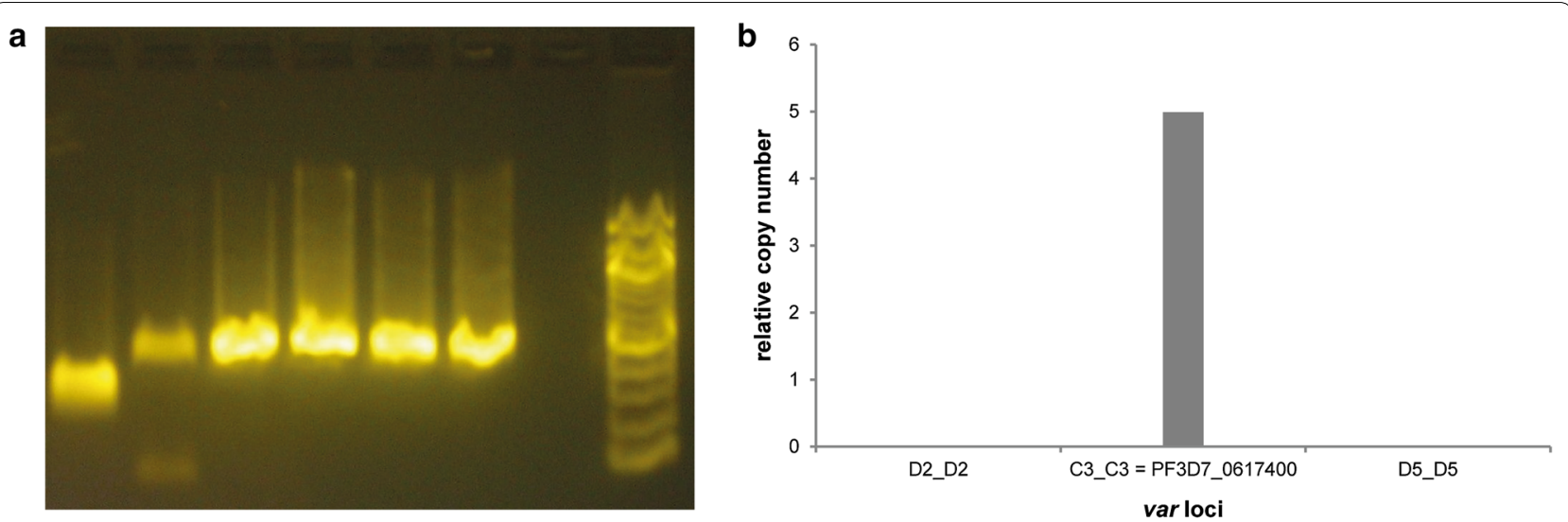

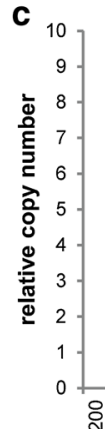

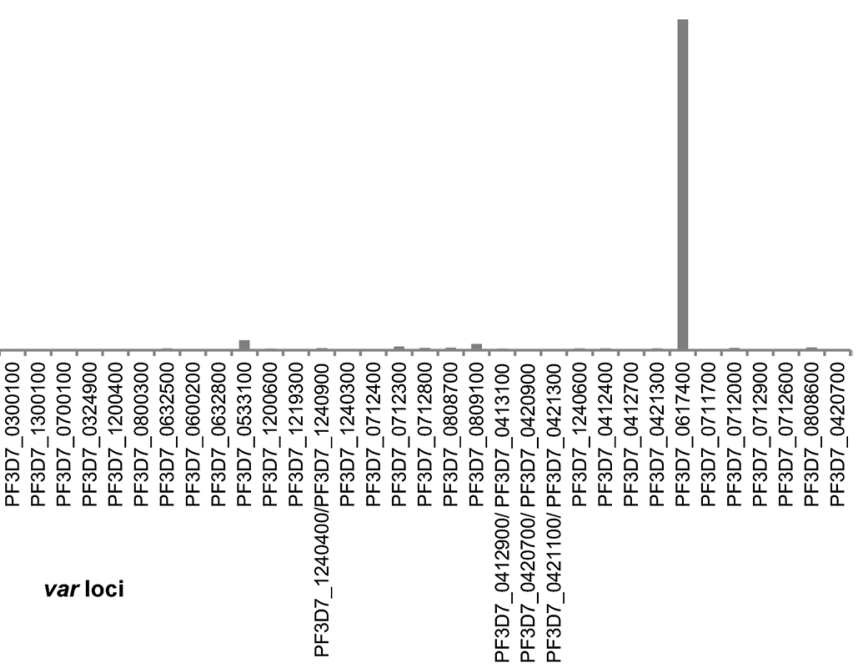

Fig. 2 a Gel electrophoresis after PCR with 6 specific PF3D7_0617400 primers (primer pairs 1,2,3,4,5,6 and a 100 bp marker) on MOA C3 cDNA reveals transcription of the entire exon 1 sequence. $\mathbf{b}$ qRT-PCR with P_C3 and two other MOA DBL primers (encoding for the dominant var genes in the other clones generated in the same limiting dilution experiment) [19] shows that PF3D7_0617400 is transcribed as the dominant var gene in MOA-C3. c qRT-PCR with NF54 specific primer set on CDNA of NF54 A3 [22] shows continued dominant transcription of PF3D7_0617400.

Transcription is quantified in relative copy numbers of the housekeeping gene arginyl tRNA synthetase in MOA C3 and NFA3

\section{Identification of PF3D7_0617400 in P. falciparum field isolates from West, Central and East Africa}

To generate a first estimate of the degree of PF3D7_0617400 conservation in P. falciparum strains, a panel of 9 additional freshly culture adapted field isolates from Central Africa (Congo, Cameroon), West Africa (Gambia, Ghana and Togo) and East Africa (Kenya and Sudan) (Table 1) were evaluated for the presence of the PF3D7_0617400 var locus and the highly conserved var2csa gene [26]. All field isolates were obtained from travellers who developed symptomatic $P$. falciparum infections after returning from Africa [21]. After successful tissue culture adaptation, DNA of the field isolates was screened by targeted PCR. Initial screening for a $3 \mathrm{~kb}$ fragment of PF3D7_0617400 detected the gene in a field isolate originating from Togo (5798) (Fig. 5a). Subsequent PCR with primers spanning the entire exon 1 (Additional file 1: Table S1) amplified identical fragments in 5798 and 3D7 and targeted Sanger sequencing of the field isolate 5798 (GenBank accession number MG507306) showed that it was $99-100 \%$ identical with the 3D7 reference sequence. The only difference in the exon 1 sequence was again an insertion of 194 bp ranging from 3722 to $3871 \mathrm{bp}$ (3721 and 3914 of the 3D7 reference sequence) (Fig. 6).

To analyse the chromosomal context of the conserved gene, Long-read Pacific Biosciences whole genome sequencing [21] of the MOA (PFGA01) and 5798 (PFTG01) parasite lines was performed. In both strains the conserved gene was located in the central cluster on 

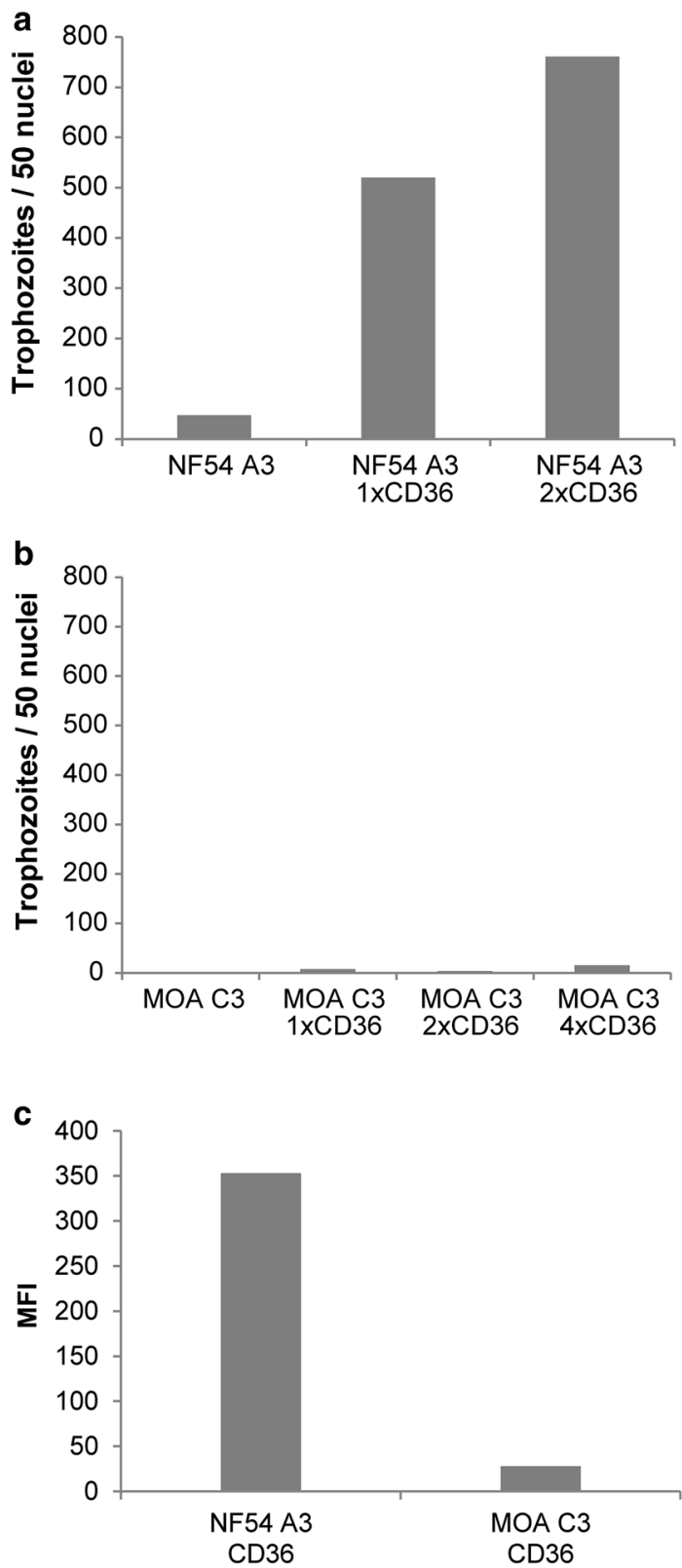

Fig. 3 CD36 binding capacity of a NF45 A3 before CD36 selection and after one and two rounds of selection and $\mathbf{b} \mathrm{MOA}$ C 3 before CD36 selection as well as after one to four rounds of selection. $\mathbf{c}$ Mean fluorescence intensity (MFI) of FACS analysis with semi-immune sera from the MOA individual [20] on NF54 A3 and MOA C3 after CD36 selection

chromosome 6 . The field isolate allele of the MOA strain was annotated as PFGA01_060022400. Interestingly the neighbouring stevor and rifin genes were also conserved in the three isolates (Fig. 6).

The whole genome sequence analysis, as well as the microsatellite analysis (see below) of PFTG01 showed that it was a mixed infection of two different parasites strains. Consequently, the de novo assembly process generated two different alleles of the central cluster on chromosome 6 (as well as for all other variable parts of the genome). The allele carrying the central cluster that is identical with the PF3D7 central cluster is located on contig PFTG01_00_33.embl.

\section{Conservation of PF3D7_0617400 and PfGA01_060022400 in a global $P$. falciparum population from Africa and Asia}

To evaluate if the presence of PF3D7_0617400/ PFGA01_060022400 in 2 out of 10 field isolates reflected the conservation of this locus in natural $P$. falciparum populations, a recently described population of 714 whole genome sequenced parasites from Africa and South East Asia [15] was screened for the presence of the PFGA01_060022400 and PF3D7_0617400. PFGA01_060022400 was detected at full length and $>95 \%$ identity in 36 of the 714 fully sequenced isolates. PFGA01_060022400 was detected at equal frequency in parasites from Africa and Asia clearly confirming the conservation of this locus in natural $P$. falciparum populations on different continents (Table 2). Interestingly, the conservation of PFGA01_060022400 was in the same order of magnitude as the conservation of PFDd2_070015900, a var gene located in the P. falciparum chloroquine resistance transporter ( $p f c r t$ ) associated genetic sweep on chromosome 7, that was detected in 33 isolates of the global P. falciparum population.

In contrast to PFGA01_060022400, the full length allele of PF3D7_0617400 was only detected in 3 isolates. However, when fragments between $3,5 \mathrm{~kb}$ and $500 \mathrm{bp}$ were analysed the prevalence of the two alleles was similar and increased from 48 to 139 isolates (Table 3).

Next, the conservation of the different parts of exon 1 of PFGA01_060022400 was analysed. This revealed that the first part of exon 1, corresponding to the DBLa0.21, CIDRa2.1 and DBLb4 (0-approximatley $3700 \mathrm{bp}$ ) were less conserved than the second part of exon 1 (approximately 3800-5800 bp) corresponding to the CIDRb1 and DBLd1 (Fig. 7). In contrast, a var gene (PfDd2_070015900) located in the pfcrt associated genetic sweep on chromosome 7 was equally conserved along the entire exon1 (Fig. 7). Furthermore, a SNP analysis in 19 isolates carrying PFGA01_060022400 showed no evidence of a genetic sweep in the chromosomal area surrounding the central cluster on chromosome 6 (Additional file 4: Fig. S2).

An analysis of conservation of all 3D7 var genes revealed that, except for the pseudogene varlcsa, PF3D7_0617400 was the most conserved 3D7 var gene, although fragments of several other var genes were also conserved to a lesser degree in the global 


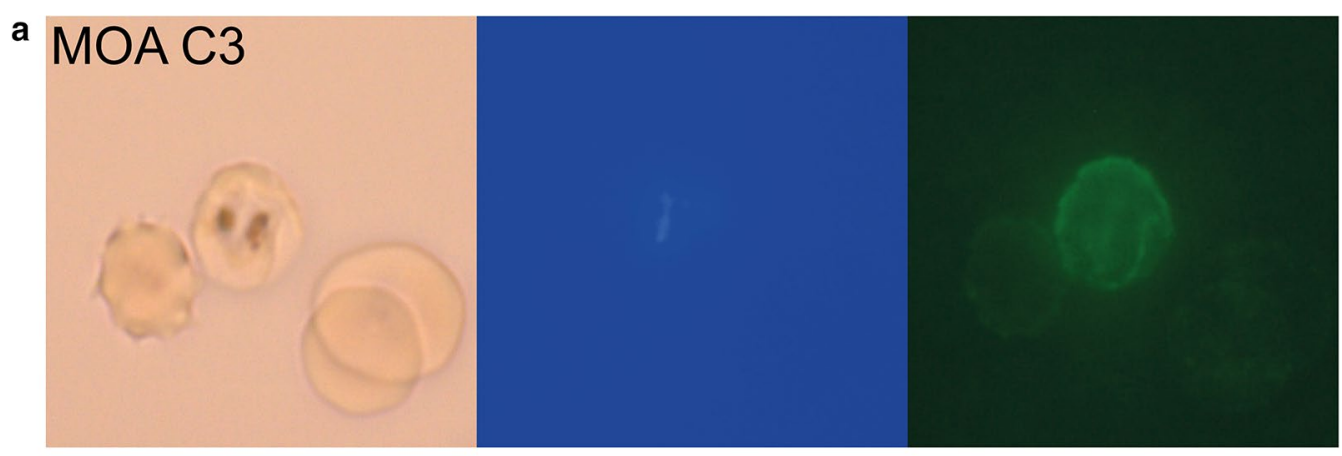

b NF54 A3

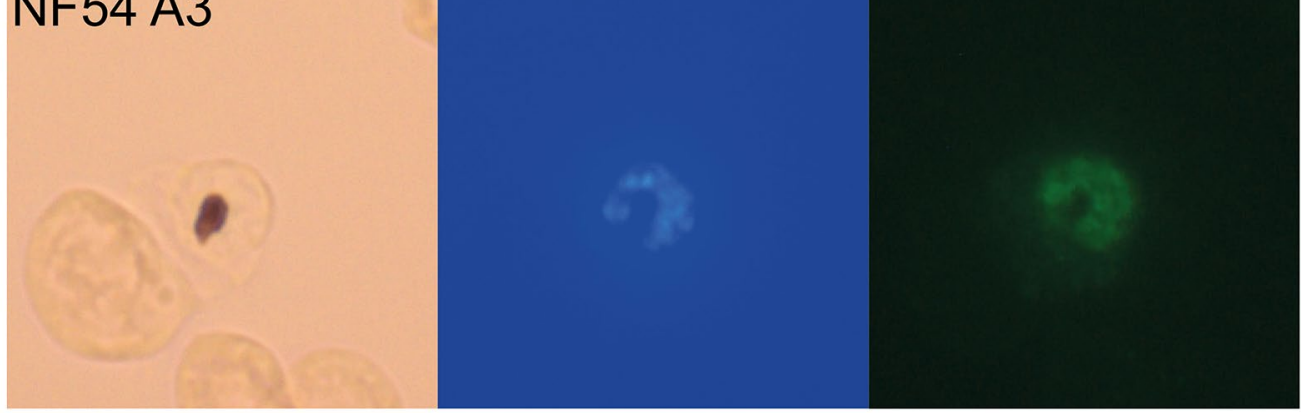

c Delta MOA D2

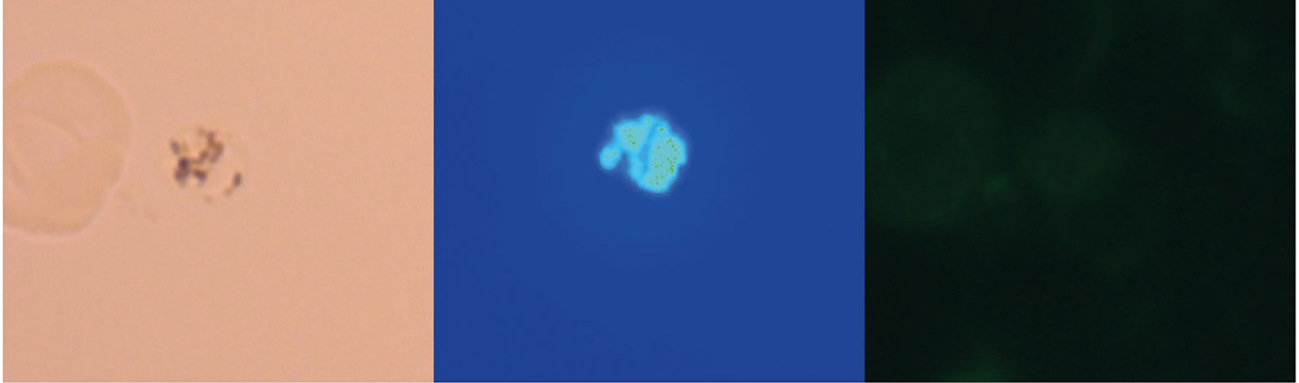

Fig. 4 Immunofluorescence with a specific antibody against the CIDRa2.1 of PF3D7_0617400 shows expression in MOA C3 and NF54 A3 but not in the field isolate $\triangle M O A D 2:$ a MOA C3, b NF54 A3, c $\triangle$ MOA D2 (propagated without blasticidin pressure). The first images displays light microscopy, the second image identifies parasitized RBCs by Hoechst DNA staining (blue) and the third image displays IFA with mouse anti-CIDRa2.1-PF3D7_0617400 antibody and GFP. Note the anti-CIDRa2.1 antibodies signal in MOA C3 and NF54 A3. The D2 DBL expressed in $\triangle$ MOA D2 [20] is not detected by the antibody

Table 1 Field isolate overview: isolate numbers, country of origin

\begin{tabular}{ll}
\hline Isolate No. & Country of origin \\
\hline 5259 & Congo \\
12295 & Cameroon \\
5420 & Kenya \\
12480 & Kenya \\
5798 & Togo \\
3256 & Togo, Ghana \\
3324 & Kenya \\
6022 & The Gambia \\
6210 & Sudan \\
MOA C3 & Gabon \\
\hline
\end{tabular}

population of P. falciparum parasites (Additional file 5: Fig. S3). Together the data showed that individual parts of PFGA01_060022400/PF3D7_0617400 and other var genes exhibited less recombination than the remainder of the gene family.

\section{Comparative analysis of microsatellite (MS) and var2csa genetic diversity}

To further investigate the hypothesis that recombination of individual shorter var sequences might be limited, the genetic diversity of 57 microsatellite markers (average length approximately $160 \mathrm{bp}$ ) and two short var2csa 


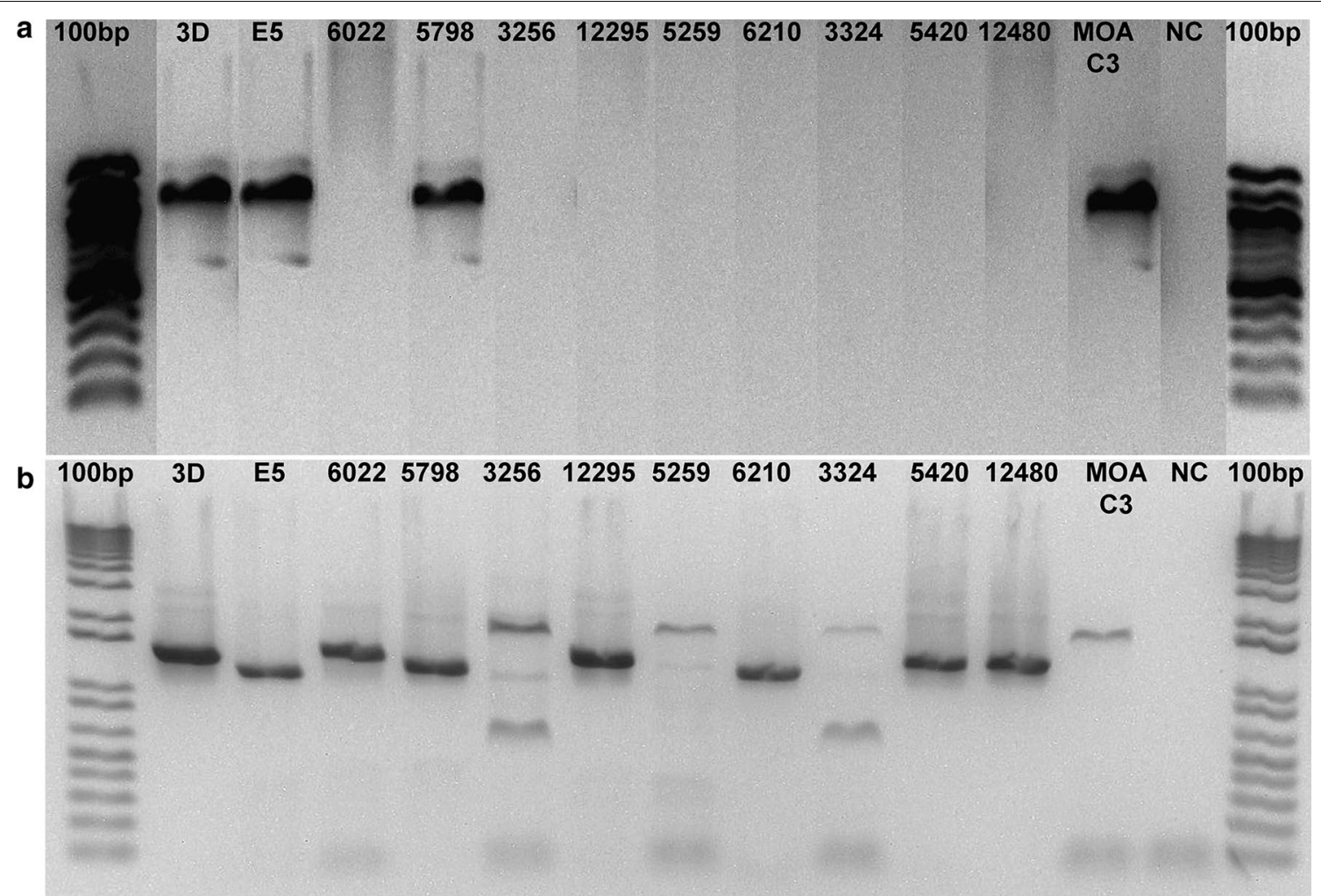

Fig. 5 PF3D7_0617400 and var2csa in field isolates. a Gel electrophoresis of PCR fragments of PF3D7_0617400 on all field isolates and controls. PCR fragments of PF3D7_0617400 amplified (primer pair POF + P0.1R) on two field isolated (5798, MOA C3) and on the NF54 clones 3D7 and E5 (positive controls). $\mathrm{NC}=$ negative control. The gel was digitally rearranged to match the gel shown in $\mathbf{b}$. $\mathbf{b}$ Gel electrophoresis of PCR fragments of var2csa on all field isolates and controls. PCR fragments of var2csa amplified by one primer pair (10F and 75R, [5]) on all field isolates and on the NF54 clones 3D7 and E5 (positive controls). $\mathrm{NC}=$ negative control

fragments (156 bp and 141 bp length) was compared in the original population of 10 field isolates.

A total of 57 MS distributed across the 14 P. falciparum chromosomes (three to four MS per chromosome) (Fig. 8a) were typed for each field isolate. Allele analysis of the 57 MS across the 10 field isolates revealed the presence of a unique allele in each strain at the vast majority of MS. Fragment length diversity was therefore high across all microsatellites with an expected heterozygosity $\left(\mathrm{H}_{\mathrm{e}}\right)$ ranging between 0,53 and 0,98 and an average $\mathrm{H}_{\mathrm{e}}$ across all microsatellites of 0.8 (Fig. 8b) (Additional file 2: Table S2).

The expected heterozygosity $\left(\mathrm{H}_{\mathrm{e}}\right)$ of var2csa was calculated for two fragments, a highly conserved (bp 34023558) (156 bp) (fragment I) as well as a variable region (bp 2664-2805) (141 bp) (fragment II). Fragment I had the same length and sequence in all 10 isolates and the $\mathrm{H}_{\mathrm{e}}$ was 0 . Fragment II differed in length and sequence in all field isolates and had a $\mathrm{H}_{e}$ of 0.86 . Sequence analysis of this fragment revealed deletions, insertion as well as single base pair substitutions as the most common differences (data not shown). Dot plot comparison of the
$57 \mathrm{MS} \mathrm{H}_{\mathrm{e}} \mathrm{s}$ and the two var2csa fragments $\mathrm{H}_{\mathrm{e}} \mathrm{s}$ showed that fragment II was as diverse as the majority of the MS whereas fragment I was highly conserved (Fig. 9).

\section{Discussion}

In this work, a conserved var gene PFGA01_060022400/ PF3D7_0617400 (previously annotated as MAL6P1.252 /PFF0845c) is identified in parasites from Africa and Asia. PFGA01_060022400/ PF3D7_0617400 is located in the central cluster of chromosome 6 . The predicted PfEMP1 domain structure of PFGA01_060022400/ PF3D7_0617400 consists of a DBLa0.21, a CIDRa2.1, a DBLb4, a CIDRb1 and aDBLd1 domain. The only difference between the 3D7 and the field isolate allele is a small insertion of approximately $190 \mathrm{bp}$ located between the DBLb4 and CIDRb1.

The CIDR a2.1 [31] of PFGA01_060022400/ PF3D7_0617400 possesses the recently described hydrophobic pocket that is responsible for PfEMP1 binding to the CD36 receptor [18]. Consistent with this, NF54 parasites transcribing PF3D7_0617400 bound efficiently to CD36 receptors on human melanoma cells, showing that 


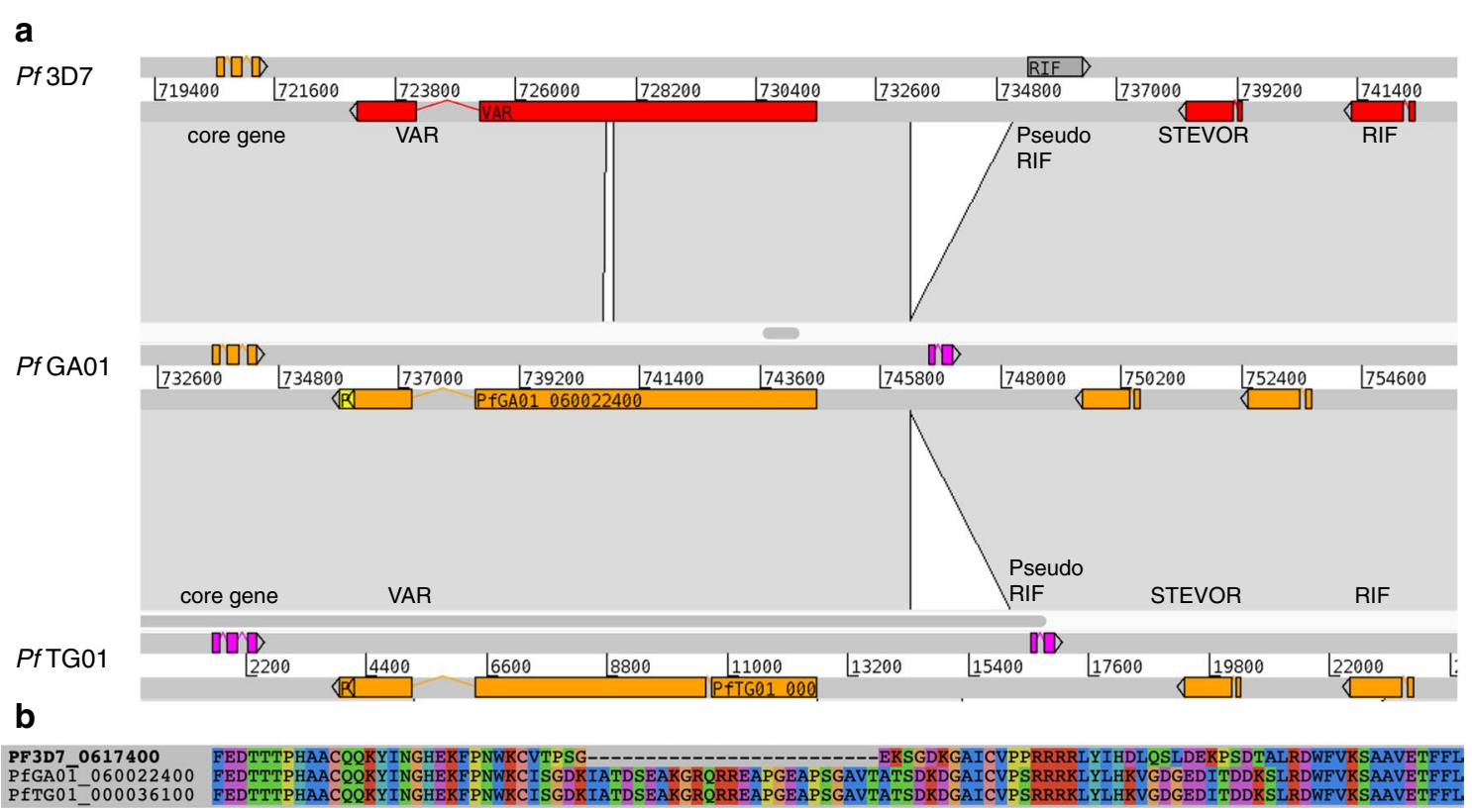

Fig. 6 a ACT program comparative analysis of the central cluster of chromosome 6 in the 3D7 genome strain, MOA C3 (PFGA01) (middle part of figure) and 5798 (PFTG01) (lower part of figure), note that the stevor and rifin genes located downstream of PF3D7_0617400 are also conserved in the 3 strains. $\mathbf{b}$ Amino acid alignment depicting the amino acid sequence difference between 3D7 and the two field isolates

Table 2 Conservation of PFGA01_060022400 in 714 isolates from Africa and Asia

\begin{tabular}{lll}
\hline Country of origin & Analysed isolates & $\begin{array}{l}\text { PFGA01_060022400 } \\
\text { >3500 bp }\end{array}$ \\
\hline The Gambia & 60 & 9 \\
Malawi & 60 & 8 \\
Laos & 60 & 6 \\
Cambodia & 60 & 6 \\
Mali & 60 & 4 \\
Ghana & 60 & 4 \\
Congo & 60 & 3 \\
Vietnam & 59 & 3 \\
Thailand & 60 & 3 \\
Guinea & 60 & 2 \\
Kenya & 55 & 1 \\
\hline
\end{tabular}

the expressed PfEMP1 exhibits the promiscuous CD36 binding phenotype. Although the hydrophobic pocket is present in virtually all CIDRa2-6, the sequence similarity is generally very low, as is the overall sequence similarity across the global population of CIDRa2-6 [32]. This raises the question which binding phenotype might be conferred by the remainder of the PFGA01_060022400/ PF3D7_0617400 domains.

Metwally et al. [31] conducted a comprehensive cytoadhesion analysis of the 3D7 laboratory strain on
Table 3 Conservation of fragments from 5000 to 500 bp in 714 isolates from Africa and Asia

\begin{tabular}{lcc}
\hline Size of fragment & PFGA01_060022400 & PF3D7_0617400 \\
\hline$>5000 \mathrm{bp}$ & 36 & 3 \\
$>4000 \mathrm{bp}$ & 42 & 3 \\
$>3500 \mathrm{bp}$ & 48 & 41 \\
$>3000 \mathrm{bp}$ & 50 & 44 \\
$>2500 \mathrm{bp}$ & 51 & 45 \\
$>2000 \mathrm{bp}$ & 76 & 108 \\
$>1500 \mathrm{bp}$ & 92 & 129 \\
$>1000 \mathrm{bp}$ & 94 & 131 \\
$>500 \mathrm{bp}$ & 100 & 139 \\
\hline
\end{tabular}

CHO-745 WT cells and in CHO-745 cells expressing recombinant CD36, Intracellular Adhesion Molecule 1 (ICAM) 1, P-selectin, E-selectin, CD9 and CD151. 3D7 parasites showed strong upregulation of PF3D7_0617400 transcription after selection on all cell types. The strongest upregulation was seen after binding selection on Chinese Hamster Ovary (CHO) wild type (WT) cells with PF3D7_0617400 being the only significantly upregulated var gene ( $84 \%$ of the total var gene signal). Together these data suggest that PF3D7_0617400 is able to bind to a yet unidentified receptor on CHO WT cells as well as CD36, ICAM, P-selectin, E-selectin, CD9 and CD15. Synergistic binding to multiple receptors has [33] been shown to 


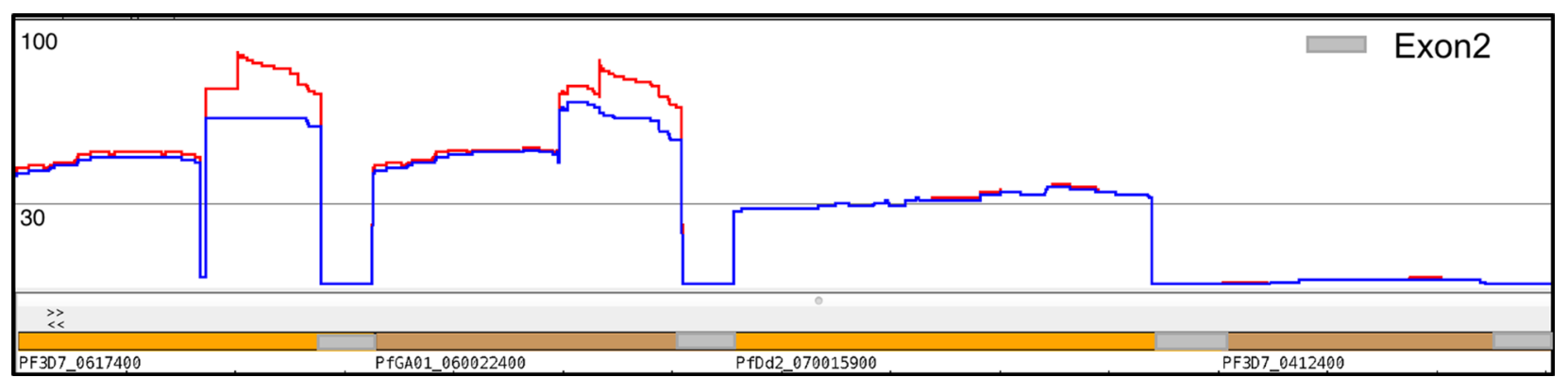

Fig. 7 Exon 1 conservation of PF3D7_0617400, PFGA01_060022400, PFDd2_070015900 and PFD3D7_0412400 in a global population of parasites. The amount of sharing of 4 var genes among a population of 714 parasites from Africa and Asia [15]. Blue parts correspond to fragments $>2000$ bp, red parts correspond to fragments $>500 \mathrm{bp}$. The $y$ axis shows the number of isolates carrying the respective fragments. Note that the second part of exon I is conserved to a higher degree than the first part. The small insertion that marks the difference between PF3D7_0617400 and PFGA01_060022400 can be appreciated. Note that PFDd2_070015900 is conserved along the entire exon1, consistent with a location within the pfcrt genetic sweep. PFD3D7_0412400 is shown as an example of a nonconserved UpsC 3D7 var gene. Exon 2 sequences were excluded from the analysis

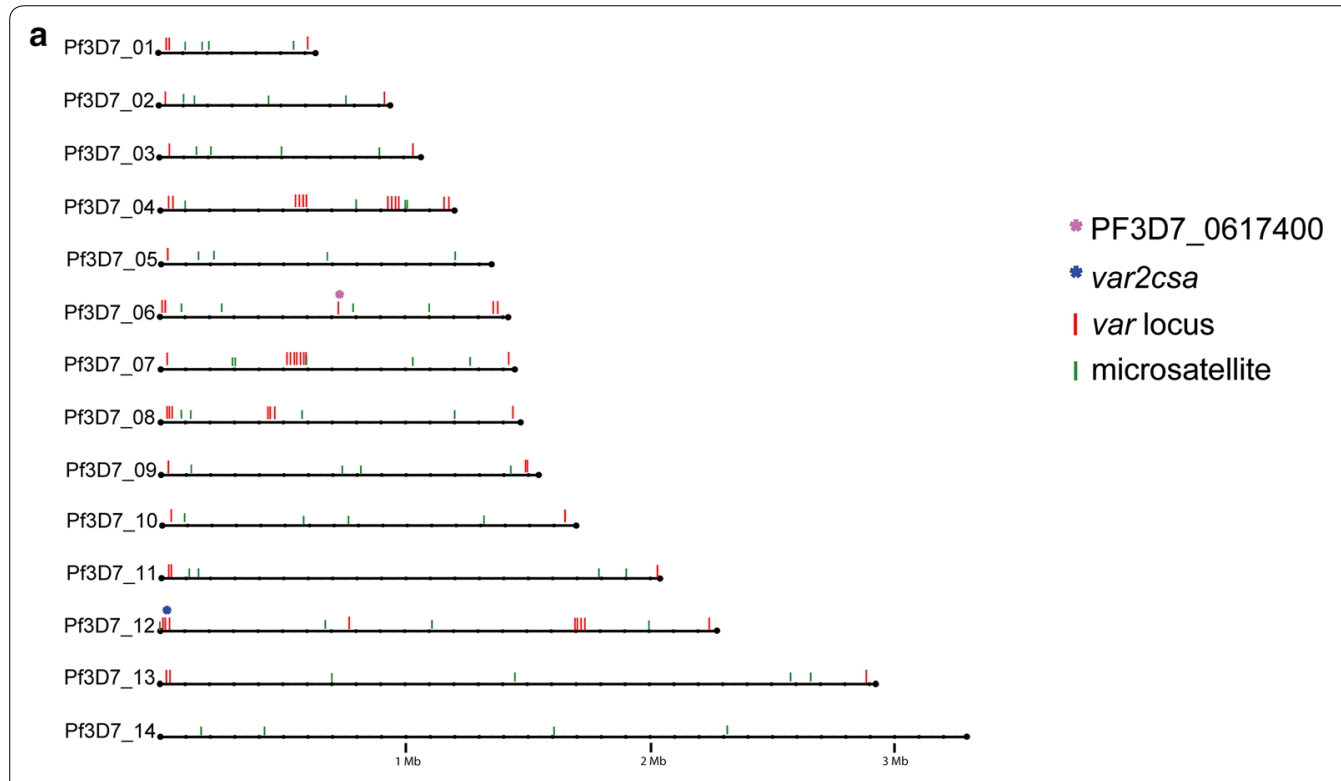

b

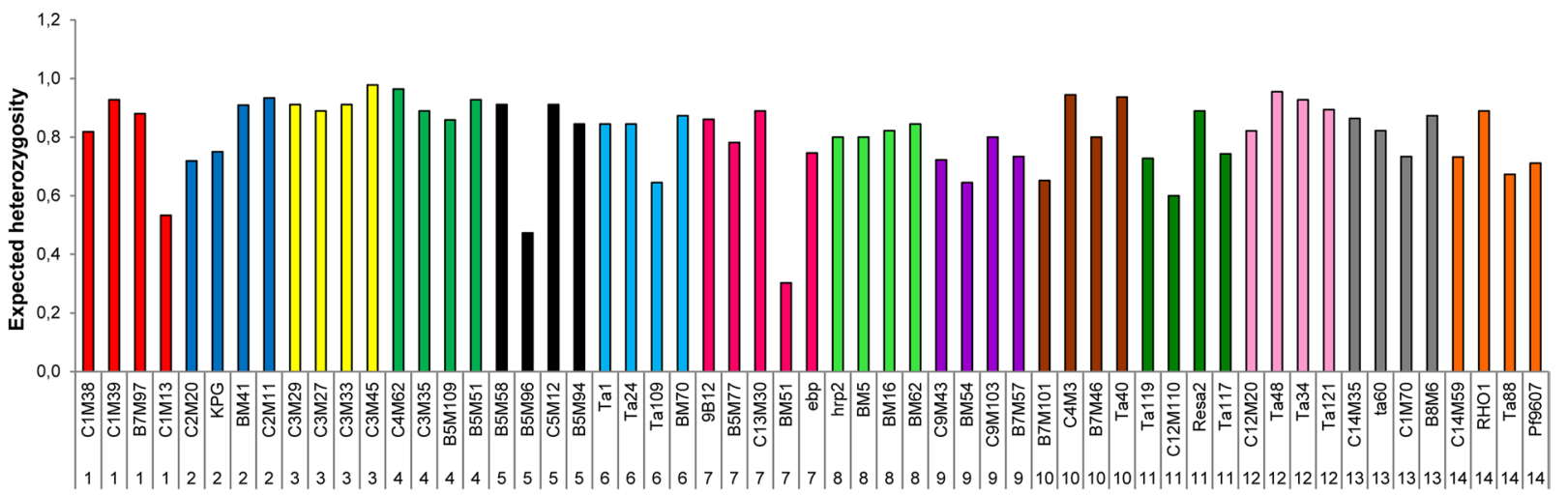

Fig. 8 Microsatellite position and genetic diversity a 14 chromosomes of $P$. falciparum with positions of var genes indicated in red and positions of microsatellites indicated in green. $\mathbf{b}$ Observed $\mathrm{H}_{\mathrm{e}}$ of microsatellite loci on all 14 chromosomes of $P$. falciparum across all field isolates. $0=$ no diversity, $1=$ max. diversity 


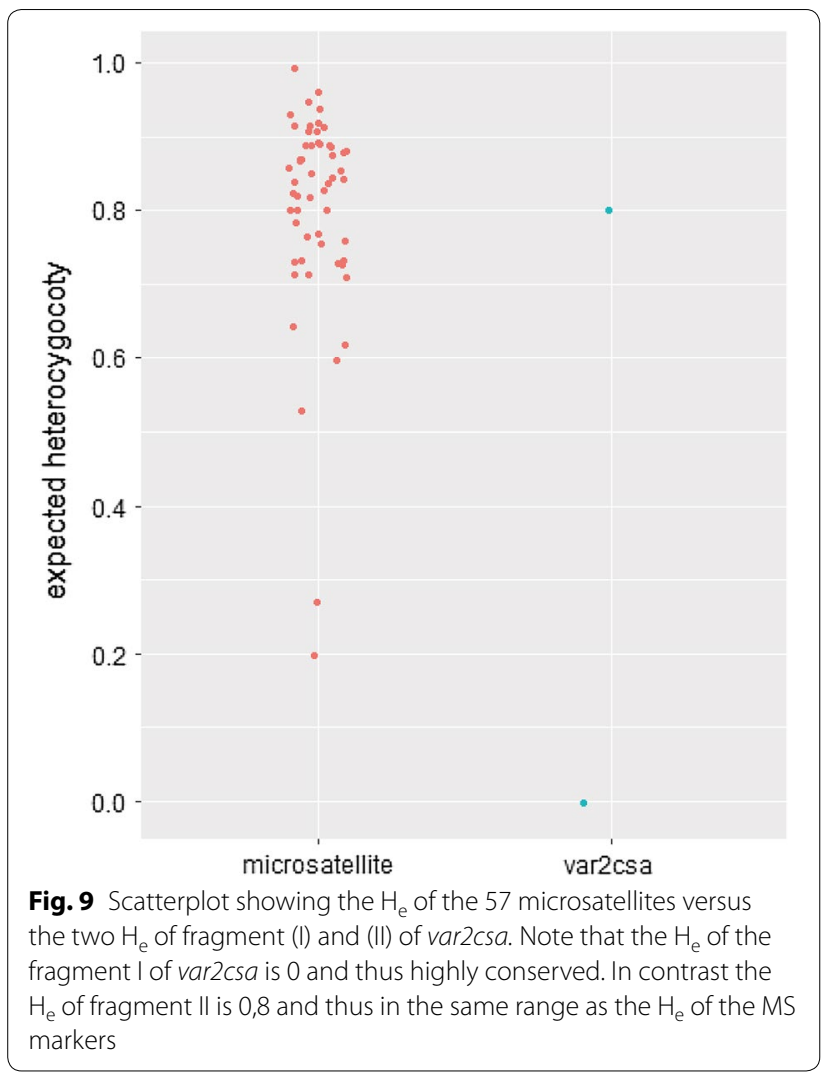

confer more efficient binding providing a potential explanation why PF3D7_0617400/ PFGA01_060022400 might confer a selective advantage.

The field isolate clone MOA C3 transcribed PFGA01_060022400 in a very stable fashion yet it was not possible to increase the low binding capacity of the strain to human melanoma cells expressing recombinant CD36. The MOA parasite line was originally obtained from a chronic asymptomatic infection that lasted for more than 90 days. The MOA parasites had therefore been under strong selective pressures prior to tissue culture adaptation. A previous phenotypic analysis of 19 clones from the MOA bulk culture showed that immune recognition of MOA clones did not correlate with var gene transcription [20]. Here, these observations are extended by phenotypic profiling of an NF54 and a MOA clone transcribing the same var gene. CD36 selected NF54 A3 and MOA C3 parasites exhibited a marked difference in surface recognition signal and CD36 binding, yet the corresponding PfEMP1 was detected by immunofluorescence assay (IFA) in both cell lines. This suggests a difference in PfEMP1 display between the two cell lines. Several investigations have reported that PfEMP1 expression is influenced by semi-immunity [20, 34-36] and Hoo et al. [37] have shown recently that the duration of replication in the human host has a strong impact on the P. falciparum transcriptome. It is thus tempting to hypothesize that the prolonged intra-host replication of the MOA parasites selected for parasites with reduced PfEMP1 display to allow the submicroscopic parasitaemia of chronic infections. It is conceivable that expression of a PfEMP1 variant with synergistic binding to multiple receptors provides a selective advantage during chronic infections. Future investigations with culture adapted parasites from semi-immune individuals are necessary to further address this question.

PFGA01_060022400 was identified in 37 isolates of a recently characterized global population of 714 fully sequenced isolates. A previous analysis of this population had identified several var genes with fragments > $3.5 \mathrm{~kb}$ that were shared at $99 \%$ percent identity in up to 55 isolates. These genes are located on chromosomes 7, 4, and 8 in the areas of drug resistance associated selective sweeps. An additional conserved var gene was located in a telomeric genetic sweep of chromosome 6. In contrast the parasite isolates carrying PFGA01_060022400/ PF3D7_0617400 showed no evidence of a genetic sweep in chromosomal areas flanking the conserved locus in the central cluster of chromosome 6 . This suggests that PFGA01_060022400/PF3D7_0617400 might provide some independent "fitness advantage". The population analysis also showed that the DBLb4 and CIDRb1 sequences were present in more isolates than the DBLa0.21, CIDRa2.1, DBLb4, CIDRb1 sequences, indicating that fragments of the gene are conserved to different degrees in the global $P$. falciparum population, suggesting that individual sequence blocs might be under "positive selection".

To further evaluate this hypothesis, the genetic diversity of a highly variable and highly conserved part of the DBL2x domain of the var2csa gene and the genetic diversity of 57 MS [26] were compared in the original population of 10 field isolates. As expected the genetic diversity of MS was high (0.76-0.8) and in the range of previously reported values for MS genetic diversity [27, 38-40]. Strikingly, the variable DBL2x part exhibited the same average genetic diversity $\left(\mathrm{H}_{\mathrm{e}}=0.8\right)$ as the MS, suggesting that sequence variation in these parts of the gene have no deleterious effect on parasite fitness. In contrast the genetic diversity of the conserved DBL2x fragment was 0 and indeed the sequence was completely identical across all isolates. These data are consistent with a recent analysis of the var2csa gene in a global population of $>2000$ isolates that showed a high sequence diversity along the DBL2x but a high degree of conservation in the area corresponding to the conserved fragment [41]. 
While var2csa is present in every parasite, the full length PFGA01_060022400/ PF3D7_0617400 was only detected in approximately $5 \%$ of field isolates. PFGA01_060022400 /PF3D7_0617400 is located in the central cluster of chromosome 6 and belongs to the UpsC subclass of var genes. UpsC var genes have been shown to be preferentially transcribed during long term in vitro culture and during chronic asymptomatic infections [19, 22, 42]. The binding phenotype of PFGA01_060022400 /PF3D7_0617400 may, therefore, be important to establish chronic asymptomatic infections. If receptor binding is indeed responsible for the conservation of PFGA01_060022400 /PF3D7_0617400 this would imply that other var genes must confer the same binding phenotype. The recent global analysis of the var gene family by Otto et al. [15] showed that UpsC genes are the most conserved among the var gene classes which could be consistent with a common binding phenotype mediated by UpsC var genes. In summary, the data reported here suggests that individual PfEMP1 binding phenotypes may limit the sequence diversity of individual members of the var gene family.

\section{Conclusion}

In this study, a conserved var gene on chromosome 6 is identified in parasites from Africa and Asia. This indicates that some var loci appear to be under purifying selection because they provide a selective advantage. The conserved var gene binds to multiple receptors, a phenotype that might increase parasite fitness during chronic infections. Interestingly, direct phenotypic comparison of a laboratory and a field isolate from a chronic infection expressing the locus showed marked differences in receptor binding suggesting a difference in PfEMP1 display.

\section{Supplementary information}

Supplementary information accompanies this paper at https://doi. org/10.1186/s12936-020-03257-x.

\section{Additional file 1: Table S1. Exon 1 Primer pairs designed for targeted} Sanger sequencing of PF3D7_0617400.

Additional file 2: Table S2. base pair length and $\mathrm{H}_{\mathrm{e}}$ of $57 \mathrm{MS}$ across the population of field isolates.

Additional file 3: Fig. S1. Quantitative transcriptional analysis with RTPCR. a) Transcriptional profile of the var gene family after CD36 binding selection of NF54 A3 shows a strong increase in transcriptional signal of PFD 0617400. b) Transcriptional profile of MOA C3 after CD36 binding selection of NF54 A3 shows no change in transcriptional signal of PFD_0617400.

Additional file 4: Fig. S2. SNP analysis of chromosome 6 of 19 parasites carrying PFGA01_060022400. The area of chromosome 6 flanking the central cluster is shown. The position of PFGA01_060022400 is depicted by the vertical red line. SNPs are indicated by bars of different colour. There is no evidence of genetic sweep in the areas flanking the locus.
Additional file 5: Fig. S3. Analysis of 3D7 var gene conservation within the global population of 714 parasites. Fragments of $>3000 \mathrm{bp}$ that are conserved within the global population are depicted. The red square identifies PF3D7_0617400.

\section{Abbreviations}

CD: Cluster of differentiation; iRBCs: Infected red blood cells; PfEMP1: Plasmodium falciparum erythrocyte membrane protein 1; EPCR: Endothelial protein C receptor; Ups: Upstream; DC: Domain cassette; DBL: Duffy binding like; CIDR: Cysteine rich interdomain; CSA: Chondroitin sulfate A; PCR: Polymerase chain reaction; ORF: Open reading frame; qPCR: Quantitative PCR; pfcrt: Plasmodium falciparum chloroquine resistance transporter; MS: Microsatellite; $\mathrm{H}_{\mathrm{e}}$ : Expected heterozygosity; ICAM 1: Intracellular adhesion molecule 1; CHO: Chinese Hamster Ovary; WT: Wilde type; IFA: Immunofluorescence assay; MFI: Mean fluorescence intensity.

\section{Acknowledgements}

We thank Mrs. Andrea Weierich for her support in performing the PCR analysis of the field isolates and for assembling the PF3D7_0617400 sequence of the MOA C 3 and 5794 parasite strains. We thank Emma Frank for her assistance in the preparation of the final version of the manuscript. We thank Thomas Lavstsen for facilitating the CIDRa2.1 antibody production.

\section{Authors' contributions}

SD conducted the PCR and the targeted Sanger sequencing analysis of PF3D7_0617400 as well as the transcriptional analysis. SD and EB conducted the IFA analysis. CE generated the primer pairs P1-6, performed the initial amplification experiments and conducted the CD36 binding assays. EB performed the MS genetic analysis. LT generated the anti-PFD_0617400 antibodies. TO performed the long-read Pacific Biosciences whole genome sequencing and the subsequent analysis. PK provided conceptual advice. MF designed the project. SD, EB and MF wrote the manuscript. All authors read and approved the final manuscript.

\section{Funding}

This work was supported by the German Israeli Foundation (to C.E., SD. and M.F.) and the German ministry for education and research (BMBF) (Grant Nr. 01KA110) (to E.B. and M.F.). We acknowledge support by Open Access Publishing Fund of University of Tübingen.

\section{Availability of data and materials}

The datasets supporting the conclusions of this article are included within the article and its additional files.

\section{Ethics approval and consent to participate}

Ethical approval for characterization of the long-term chronic infection of MOA was obtained from the local ethics committee in Lambarene as previously described (Enderes et al. [19] and Bruske et al. [20]). Field isolates obtained in Tübingen were generated in the process of routine laboratory diagnosis and thus didn't require individual ethical approval.

\section{Consent for publication}

Not applicable.

\section{Competing interests}

The authors declare that they have no competing interests.

\section{Author details}

${ }^{1}$ Institute of Tropical Medicine, University of Tuebingen, Wilhelmstr. 27, 72074 Tuebingen, Germany. ${ }^{2}$ Malaria Programme, Wellcome Trust Sanger Institute, Hinxton CB10 1SA, UK. ${ }^{3}$ Centre of Immunobiology, Institute of Infection, Immunity \& Inflammation, College of MVLS, University of Glasgow, Glasgow, UK. ${ }^{4}$ Centre for Medical Parasitology, Department of Immunology and Microbiology (ISIM), Faculty of Health and Medical Sciences, University of Copenhagen, 1165 Copenhagen, Denmark. ${ }^{5}$ Department of Infectious Diseases, Copenhagen University Hospital (Rigshospitalet), 2100 Copenhagen, Denmark. 
Received: 31 October 2019 Accepted: 15 May 2020

Published online: 29 May 2020

\section{References}

1. WHO. World Malaria Report 2016. Geneva, World Health Organization; 2016. http://www.who.int/malaria/publications/world-malaria-repor t-2016/report/en/. Accessed 17 Aug 2017.

2. Miller LH, Baruch DI, Marsh K, Doumbo OK. The pathogenic basis of malaria. Nature. 2002:415:673-9.

3. Smith JD, Chitnis CE, Craig AG, Roberts DJ, Hudson-Taylor DE, Peterson DS, et al. Switches in expression of Plasmodium falciparum var genes correlate with changes in antigenic and cytoadherent phenotypes of infected erythrocytes. Cell. 1995;82:101-10.

4. Baruch DI, Pasloske BL, Singh HB, Bi X, Ma XC, Feldman M, et al. Cloning the $P$. falciparum gene encoding PfEMP1, a malarial variant antigen and adherence receptor on the surface of parasitized human erythrocytes. Cell. 1995:82:77-87.

5. Salanti A, Staalsoe T, Lavstsen T, Jensen ATR, Sowa MPK, Arnot DE, et al. Selective upregulation of a single distinctly structured var gene in chondroitin sulphate A-adhering Plasmodium falciparum involved in pregnancy-associated malaria. Mol Microbiol. 2003;49:179-91.

6. Tuikue Ndam NG, Salanti A, Bertin G, Dahlbäck M, Fievet N, Turner L, et al. High level of var2csa transcription by Plasmodium falciparum isolated from the placenta. J Infect Dis. 2005;192:331-5.

7. Turner L, Lavstsen T, Berger SS, Wang CW, Petersen JEV, Avril M, et al. Severe malaria is associated with parasite binding to endothelial protein C receptor. Nature. 2013;498:502-5.

8. Su XZ, Heatwole VM, Wertheimer SP, Guinet F, Herrfeldt JA, Peterson $D S$, et al. The large diverse gene family var encodes proteins involved in cytoadherence and antigenic variation of Plasmodium falciparuminfected erythrocytes. Cell. 1995;82:89-100.

9. Gardner MJ, Hall N, Fung E, White O, Berriman M, Hyman RW, et al. Genome sequence of the human malaria parasite Plasmodium falciparum. Nature. 2002;419:498-511.

10. Dzikowski R, Frank M, Deitsch K. Mutually exclusive expression of virulence genes by malaria parasites is regulated independently of antigen production. PLoS Pathog. 2006;2:e22.

11. Kyes SA, Kraemer SM, Smith JD. Antigenic variation in Plasmodium falciparum: gene organization and regulation of the var multigene family. Eukaryot Cell. 2007:6:1511-20.

12. Lavstsen T, Salanti A, Jensen ATR, Arnot DE, Theander TG. Sub-grouping of Plasmodium falciparum 3D7 var genes based on sequence analysis of coding and non-coding regions. Malar J. 2003;2:27.

13. Rask TS, Hansen DA, Theander TG, Gorm Pedersen A, Lavstsen T. Plasmodium falciparum erythrocyte membrane protein 1 diversity in seven genomes-divide and conquer. PLoS Comput Biol. 2010;6:e1000933.

14. Barry AE, Leliwa-Sytek A, Tavul L, Imrie H, Migot-Nabias F, Brown SM, et al. Population genomics of the immune evasion (var) genes of Plasmodium falciparum. PLoS Pathog. 2007;3:e34.

15. Otto TD, Assefa SA, Böhme U, Sanders MJ, Kwiatkowski D, Pf3k consortium, et al. Evolutionary analysis of the most polymorphic gene family in falciparum malaria. Wellcome Open Res. 2019;4:193.

16. Hamilton WL, Claessens A, Otto TD, Kekre M, Fairhurst RM, Rayner JC, et al. Extreme mutation bias and high AT content in Plasmodium falciparum. Nucleic Acids Res. 2016:45:1889-901.

17. Bopp SER, Manary MJ, Bright AT, Johnston GL, Dharia NV, Luna FL, et al. Mitotic evolution of Plasmodium falciparum shows a stable core genome but recombination in antigen families. PLoS Genet. 2013;9:e1003293.

18. Hsieh F-L, Turner L, Bolla JR, Robinson CV, Lavstsen T, Higgins MK. The structural basis for CD36 binding by the malaria parasite. Nat Commun. 2016;7:12837

19. Enderes C, Kombila D, Dal-Bianco M, Dzikowski R, Kremsner P, Frank M. Var gene promoter activation in clonal Plasmodium falciparum isolates follows a hierarchy and suggests a conserved switching program that is independent of genetic background. J Infect Dis. 2011;204:1620-31.

20. Bruske El, Dimonte S, Enderes C, Tschan S, Flötenmeyer M, Koch I, et al. In vitro variant surface antigen expression in Plasmodium falciparum parasites from a semi-immune individual is not correlated with var gene transcription. PLoS ONE. 2016;11:e0166135.
21. Otto TD, Böhme U, Sanders M, Reid A, Bruske El, Duffy CW, et al. Long read assemblies of geographically dispersed Plasmodium falciparum isolates reveal highly structured subtelomeres. Wellcome Open Res. 2018;3:52.

22. Frank M, Dzikowski R, Amulic B, Deitsch K. Variable switching rates of malaria virulence genes are associated with chromosomal position. Mol Microbiol. 2007;64:1486-98

23. Rottmann M, Lavstsen T, Mugasa JP, Kaestli M, Jensen ATR, Müller D, et al. Differential expression of var gene groups is associated with morbidity caused by Plasmodium falciparum infection in Tanzanian children. Infect Immun. 2006;74:3904-11.

24. Taylor HM, Kyes SA, Harris D, Kriek N, Newbold Cl. A study of var gene transcription in vitro using universal var gene primers. Mol Biochem Parasitol. 2000;105:13-23.

25. Frank M, Dzikowski R, Costantini D, Amulic B, Berdougo E, Deitsch K. Strict pairing of var promoters and introns is required for var gene silencing in the malaria parasite Plasmodium falciparum. J Biol Chem. 2006;281:9942-52.

26. Bruske $\mathrm{E}$, Otto TD, Frank M. Whole genome sequencing and microsatellite analysis of the Plasmodium falciparum E5 NF54 strain show that the var, rifin and stevor gene families follow Mendelian inheritance. Malar J. 2018;17:376.

27. Frank M, Lehners N, Mayengue PI, Gabor J, Dal-Bianco M, Kombila DU, et al. A thirteen-year analysis of Plasmodium falciparum populations reveals high conservation of the mutant pfcrt haplotype despite the withdrawal of chloroquine from national treatment guidelines in Gabon. Malar J. 2011;10:304.

28. Carver TJ, Rutherford KM, Berriman M, Rajandream MA, Barrell BG, Parkhill J. ACT: the Artemis Comparison Tool. Bioinformatics (Oxf Engl). 2005:21:3422-3.

29. Bachmann A, Petter M, Tilly A-K, Biller L, Uliczka KA, Duffy MF, et al. Temporal expression and localization patterns of variant surface antigens in clinical Plasmodium falciparum isolates during erythrocyte schizogony. PLOS ONE. 2012;7:e49540.

30. Rupp I, Sologub L, Williamson KC, Scheuermayer M, Reininger L, Doerig C, et al. Malaria parasites form filamentous cell-to-cell connections during reproduction in the mosquito midgut. Cell Res. 2011;21:683-96.

31. Metwally NG, Tilly A-K, Lubiana P, Roth LK, Dörpinghaus M, Lorenzen S, et al. Characterisation of Plasmodium falciparum populations selected on the human endothelial receptors P-selectin, E-selectin, CD9 and CD151. Sci Rep. 2017;7:4069.

32. Smith JD, Rowe JA, Higgins MK, Lavstsen T. Malaria's deadly grip: cytoadhesion of Plasmodium falciparum-infected erythrocytes. Cell Microbiol. 2013;15:1976-83

33. Lennartz F, Adams Y, Bengtsson A, Olsen RW, Turner L, Ndam NT, et al. Structure-guided identification of a family of dual receptor-binding PfEMP1 that is associated with cerebral malaria. Cell Host Microbe. 2017:21:403-14.

34. Bachmann A, Bruske E, Krumkamp R, Turner L, Wichers JS, Petter M, et al. Controlled human malaria infection with Plasmodium falciparum demonstrates impact of naturally acquired immunity on virulence gene expression. PLoS Pathog. 2019;15:e1007906.

35. Abdi Al, Warimwe GM, Muthui MK, Kivisi CA, Kiragu EW, Fegan GW, et al. Global selection of Plasmodium falciparum virulence antigen expression by host antibodies. Sci Rep. 2016;6:19882.

36. Abdi Al, Hodgson SH, Muthui MK, Kivisi CA, Kamuyu G, Kimani D, et al. Plasmodium falciparum malaria parasite var gene expression is modified by host antibodies: Iongitudinal evidence from controlled infections of Kenyan adults with varying natural exposure. BMC Infect Dis. 2017;17:585

37. Hoo R, Bruske E, Dimonte S, Zhu L, Mordmüller B, Sim BKL, et al. Transcriptome profiling reveals functional variation in Plasmodium falciparum parasites from controlled human malaria infection studies. EBioMedicine. 2019:48:442-52.

38. Anderson TJC, Williams JT, Nair S, Sudimack D, Barends M, Jaidee A, et al Inferred relatedness and heritability in malaria parasites. Proc Biol Sci. 2010;277:2531-40.

39. Wootton JC, Feng X, Ferdig MT, Cooper RA, Mu J, Baruch Dl, et al. Genetic diversity and chloroquine selective sweeps in Plasmodium falciparum. Nature. 2002;418:320-3.

40. Anderson TJ, Su XZ, Bockarie M, Lagog M, Day KP. Twelve microsatellite markers for characterization of Plasmodium falciparum from finger-prick blood samples. Parasitology. 1999;119:113-25. 
41. Benavente ED, Oresegun DR, de Sessions PF, Walker EM, Roper C, Dombrowski JG, et al. Global genetic diversity of var2csa in Plasmodium falciparum with implications for malaria in pregnancy and vaccine development. Sci Rep. 2018;8:15429.

42. Recker M, Buckee CO, Serazin A, Kyes S, Pinches R, Christodoulou Z, et al. Antigenic variation in Plasmodium falciparum malaria involves a highly structured switching pattern. PLoS Pathog. 2011;7:e1001306.

\section{Publisher's Note}

Springer Nature remains neutral with regard to jurisdictional claims in published maps and institutional affiliations.
Ready to submit your research? Choose BMC and benefit from:

- fast, convenient online submission

- thorough peer review by experienced researchers in your field

- rapid publication on acceptance

- support for research data, including large and complex data types

- gold Open Access which fosters wider collaboration and increased citations

- maximum visibility for your research: over $100 \mathrm{M}$ website views per year

At BMC, research is always in progress.

Learn more biomedcentral.com/submissions 